\title{
Influence of fluorine doping on the microstructure, optical and electrical properties of $\mathrm{SnO}_{2}$ nanoparticles
}

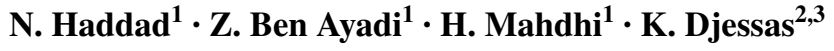

Received: 19 April 2017 / Accepted: 29 June 2017 / Published online: 4 July 2017

(c) The Author(s) 2017. This article is an open access publication

\begin{abstract}
Tin dioxide $\left(\mathrm{SnO}_{2}\right)$ is one of the most promising functional semiconductor materials; it has stimulated considerable research interest for its wide applications such as optoelectronic devices and solar cells technology. Undoped and fluorine doped tin oxide aerogel nanoparticles have been successfully synthesized by sol-gel route and dryed under supercritical conditions of ethanol. The effect of fluorine concentration on $\mathrm{SnO}_{2}$ nanoparticles properties was systematically investigated by $\mathrm{X}$-ray diffraction, scanning electron microscopy, transmission electron microscopy, Raman spectroscopy, UV-Vis-IR spectroscopy and impedance spectroscopy. Characterization data showed that both pure $\mathrm{SnO}_{2}$ and FTO aerogels have a polycrystalline tetragonal rutile structure with a highly (110) plane preferred orientation. The grain sizes in the range of 11-25 nm were obtained depending on the elaboration conditions. Raman spectra show the fundamental peaks at 477, 632, and $782 \mathrm{~cm}^{-1}$, corresponding to the $\mathrm{E}_{\mathrm{g}}, \mathrm{A}_{1 \mathrm{~g}}$, and $\mathrm{B}_{2 \mathrm{~g}}$ vibration modes, respectively, in good agreement with those for the rutile bulk $\mathrm{SnO}_{2}$. The optical band gap value of tin oxide decreases from 3.88 to $3.44 \mathrm{eV}$ with the increase of fluorine content. Impedance spectroscopy results indicate that the electrical properties are strongly dependent on
\end{abstract}

N. Haddad

haddadnesrine20@yahoo.com

1 Laboratoire de Physique des Matériaux et des Nanomatériaux appliquée à l'Environnement, Université de Gabès, Faculté des Sciences de Gabès, Cité Erriadh Manara Zrig 6072 Gabès, Tunisia

2 Laboratoire Procédés, Matériaux et Energie Solaire (PROMES-CNRS), TECNOSUD, Rambla de la thermodynamique, 66100 Perpignan, France

3 Université de Perpignan Via Domitia, 52 avenue Paul Alduy, 68860 Perpignan Cedex 9, France temperature and doping rate. These results show the strong correlation between the structural, morphological, optical and electrical properties of the samples depending on doping content which was well reflected on the quality of the nanoparticles.

\section{Introduction}

Nanomaterials and their applications have generated great interest in recent years. Side by side there is an urgent need for developing reliable synthesis methods to obtain nanoparticles with well controlled physical properties such as optical, morphologic, catalytic and electrical, which strongly depend on particle size, shape and agglomeration $[1,2]$.

Tin dioxide has attracted considerable attention due to their potential applications in optoelectronic, gas sensor, catalysis and photovoltaic devices [1]. It is a transparent conductor member due to its unique physical properties such as high electrical conductivity, transparency in the visible part of the spectrum and high chemical stability [3, 4]. The desirable properties of tin oxide $\left(\mathrm{SnO}_{2}\right)$ have led many researchers to study pure and doped $\mathrm{SnO}_{2}$ in the form of nanoparticles [5-8]. Doping with foreign impurities is an important method to enhance the properties of the nanoparticles in desirable and controllable ways. The most favored dopants are antimony which substitutes the tin cations and fluorine via substituting the oxygen atoms [9].

Usually, fluorine (F) is well a known dopant for $\mathrm{SnO}_{2}$ to obtain good quality material. It exhibits good transparency and low electrical resistivity caused by the oxygen vacancies and the substitution $\mathrm{F}$ doping [10]. Compared to the widely used indium tin oxide (ITO) and antimony doped tin oxide (ATO), fluorine doped tin oxide (FTO) is 
presented as good material due to its superior physical and chemical properties for a large range of applications like gas sensors [11-13], photocatalysts [14] and solar cells $[15,16]$. Indeed, the properties of FTO powder are affected by the preparation process. For that, the synthesis of FTO nanostructures with desired structure and morphology has become a subject of intense research in the last few years.

Thus, it is essential to develop ways for controlling their structure and surface properties. There are several techniques that have been used to prepare FTO nanoparticles and thin films such as chemical vapor deposition [17], hydrothermal [18], solvothermal [19], spray pyrolysis [20] sol-gel [21] and rf-magnetron sputtering [10]. Among these techniques, the sol-gel method attracts most of the attention to the preparation of oxide nanoparticles, with a high quality, because of its several advantages, such as excellent homogeneity, ease of controlling the doping level, ability to coat large areas, complex shapes, and low-cost processing [22].

Until now, much research has been done on the synthesis and characterization of undoped $\mathrm{SnO}_{2}$ and FTO thin films using the sol-gel technique. However, there are few works, which have been reported on synthesis of FTO nanopowder using this method, except from Gamard et al. [23] who used it on fluorine doped $\mathrm{SnO}_{2}$ nanocrystals preparation, starting from organic precursors including $\mathrm{Sn}-\mathrm{F}$ bonds, and followed by thermal treatment in air at $550^{\circ} \mathrm{C}$ to obtain nanocrystalline FTO.

Therefore, the synthesis of highly crystallized fluorine doped $\mathrm{SnO}_{2}$ through an easy method is still a challenge. The aim of this work was to develop a new process to avoid this conflict and prepare a high quality FTO nanopowder, which can be used in the future as a target material for sputtering deposition of thin films in solar cells technology.

In this study, we report a simple protocol using a slightly modified version of the conventional sol-gel method used to synthesize tin oxide aerogels. The effects of fluorine concentration on structural, morphological, optical and electrical properties of the obtained nanopowders were investigated.

\section{Experimental details}

The tin(II) chloride dehydrated $\left[\mathrm{SnCl}_{2} \cdot 2 \mathrm{H}_{2} \mathrm{O}\right.$, purity 98\%], ammonium fluorine $\left[\mathrm{NH}_{4} \mathrm{~F}\right.$, purity $\left.99.9 \%\right]$, methanol $\left[\mathrm{CH}_{4} \mathrm{O}\right.$, purity $\left.99.99 \%\right]$ and ethanol $\left[\mathrm{C}_{2} \mathrm{H}_{6} \mathrm{O}\right.$, purity 99.99\%] used in this work were analytical grade reagents, without any further purification.

Nanocrystalline FTO aerogels were successfully synthesized by sol-gel method. In a typical synthesis process $16 \mathrm{~g}$ of $\mathrm{SnCl}_{2} \cdot 2 \mathrm{H}_{2} \mathrm{O}$ was dissolved in $112 \mathrm{ml}$ of methanol. After 30 min under magnetic stirring, at room temperature, adequate quantities of $\mathrm{NH}_{4} \mathrm{~F}$, corresponding to $[\mathrm{F}] /[\mathrm{Sn}]$ atomic ratios of $0.01,0.02,0.03,0.04$ and 0.05 were added. The solution was transferred to a Pyrex glass beaker, placed in an autoclave and dried in the supercritical conditions of ethyl alcohol $\left(\mathrm{T}_{\mathrm{c}}=243^{\circ} \mathrm{C} ; \mathrm{P}_{\mathrm{c}}=63.6\right.$ bars $)$. Furthermore, the samples were annealed at $500{ }^{\circ} \mathrm{C}$ in air for $2 \mathrm{~h}$ in order to enhance their cristallinity.

The products were characterized by X-ray diffraction (XRD) using a SIEMENS D 5000 diffractometer with a CuKo radiation $(\lambda=1.5406 \AA, 40 \mathrm{kV}, 40 \mathrm{~mA})$. Elemental composition of FTO nanoparticles was analyzed using a scanning electron microscope (JEOL, JSM 5410 LV) with an energy dispersive X-ray spectroscopy (EDS) attachment. The microstructures and crystal structures of the nanoparticles were obtained using JEM-200CX transmission electron microscopy (TEM). The formation of FTO phase is further confirmed by Raman spectroscopy measurements at room temperature, using a Raman spectrometer (Renishaw inVia Reflex Spectrometer System) with a $514 \mathrm{~nm}$ Argon laser line as an excitation source. The experiments were repeated several times to confirm our result. Optical absorption properties were investigated using a Shimadzu UV3101PC Spectrophotometer in the wavelength range from 200 to $2500 \mathrm{~nm}$. The electrical measurements were performed using a two electrode configuration. The polycrystalline $\mathrm{SnO}_{2}$ samples were pressed into pellets of about $13 \mathrm{~mm}$ diameter and $2 \mathrm{~mm}$ thickness at a pressure of 20 MP a using a hydraulic press. In order to ensure ohmic contact, a thin silver film is deposited by thermal evaporation on the both sides of the pellet through a disk shaped mask. Silver wires were attached to both sides of the pellet by silver paint, giving a plate capacitor configuration. For temperature measurements, the sample is mounted in the cryostat. Electrical impedances were carried out at different temperatures using an impedance analyzer agilent $4294 \mathrm{~A}$ in the frequency range from $40 \mathrm{~Hz}$ to $1 \mathrm{MHz}$.

\section{Results and discussion}

Figure 1 shows the XRD patterns of FTO aerogel nanoparticles with different $\mathrm{F}$ doping concentrations $(0,1,2$, 3,4 and 5 at.\%). All the observed peaks can be indexed in the tetragonal structure of $\mathrm{SnO}_{2}$ according to the JCPDS card number 005-0467. The diffractograms reveal that the structure of $\mathrm{SnO}_{2}$ remains unchanged for low-doping concentration. This suggests that the fluoride ions are fully incorporated into the tin oxide lattice, without changing the tetragonal structure. This can be explained by the fact that the ionic radius of $\mathrm{F}^{-}(1.33 \AA)$ is close to that of $\mathrm{O}^{2-}(1.32 \AA)$ [24], so the doping process can take place easily. The lattice constants a and $\mathrm{c}$ were calculated from XRD data for the (110) plane, and are given in Table 1. 


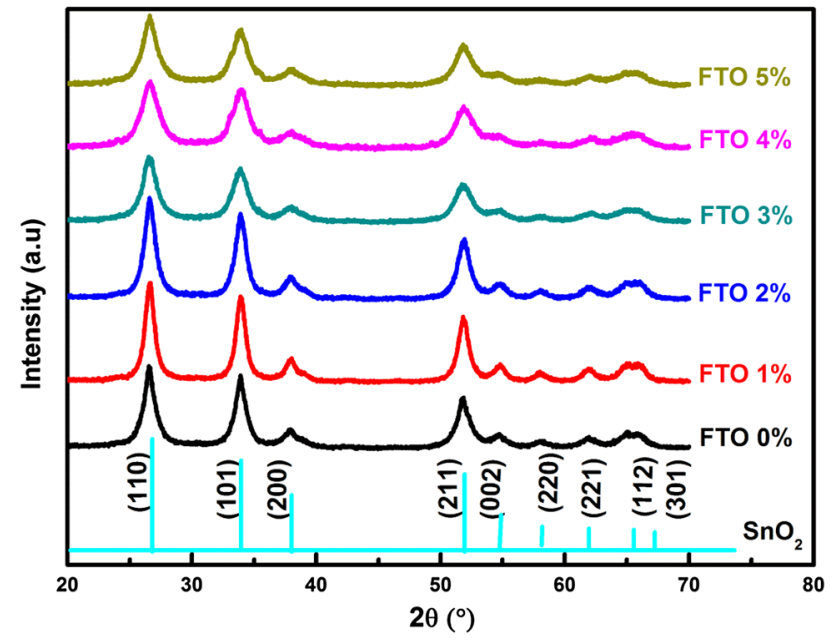

Fig. 1 X-ray patterns of FTO aerogel nanoparticles for various fluorine doping levels

$\frac{1}{d_{h k l}^{2}}=\frac{h^{2}+k^{2}}{a^{2}}+\frac{l^{2}}{c^{2}}$ where $\mathrm{h}, \mathrm{k}$ and $\mathrm{l}$ are the Miller indices, a and $\mathrm{c}$ are the lattice constants, and the inter-plane distance $\left(\mathrm{d}_{\mathrm{hkl}}\right)$ were calculated for all of the FTO nanoparticles using Bragg's diffraction equation:

$d_{h k l}=\frac{n \lambda}{2 \sin \theta}$

where $n$ is a positive integer, $\lambda$ is the $\mathrm{X}$-ray wavelength and $\theta$ is the Bragg's angle. The lattice constants calculated from the XRD patterns are found to be very similar to the $\mathrm{SnO}_{2}$ ones, i.e. $\mathrm{a}=\mathrm{b}=4.737 \AA$ and $\mathrm{c}=3.186 \AA$ [25]. The results indicate that FTO has a polycrystalline tetragonal rutile structure with a highly (110) plane preferred orientation, which agrees well with the results of previous published work by Shanshan et al. [19]. However, the comparison of the diffraction patterns of the samples revealed a variation in both intensity and broadness of the peaks. The peaks recorded for the powder obtained with 1 at. $\% \mathrm{~F}$ doped $\mathrm{SnO}_{2}$ were more intense and narrower than those of the samples prepared with F doping more than 1 at.\%. Furthermore, it is worth mentioning that the (110) diffraction
Table 1 Variation of the (110) peak positions, FWHM $(\beta)$, inter-planar distances $\left(\mathrm{d}_{\mathrm{hkl}}\right)$, grain size $(\mathrm{G})$ and lattice constants of FTO nanoparticles synthesis for different doping concentration

\begin{tabular}{llllllll}
\hline FTO (at.\%) & $(110), 2 \theta$ & $\beta\left(^{\circ}\right)$ & $\mathrm{d}_{\text {hkl }}(\AA)$ & $\mathrm{G}(\mathrm{nm})$ & $\mathrm{a}(\AA)$ & $\mathrm{c}(\AA)$ & $\mathrm{a} / \mathrm{c}$ \\
\hline 0 & 26.66 & 0.3490 & 3.341 & 23.40 & 4.736 & 3.185 & 1.486 \\
1 & 26.62 & 0.3245 & 3.345 & 25.16 & 4.730 & 3.183 & 1.486 \\
2 & 26.60 & 0.3556 & 3.348 & 22.95 & 4.734 & 3.184 & 1.486 \\
3 & 26.58 & 0.5118 & 3.358 & 15.94 & 4.737 & 3.186 & 1.486 \\
4 & 26.57 & 0.6143 & 3.360 & 13.28 & 4.751 & 3.179 & 1.494 \\
5 & 26.56 & 0.7872 & 3.365 & 11.72 & 4.758 & 3.175 & 1.498 \\
\hline
\end{tabular}

Fig. 2 Evolution of FHWM and grain size $(\mathrm{G})$ of the FTO nanoparticles for different doping concentrations

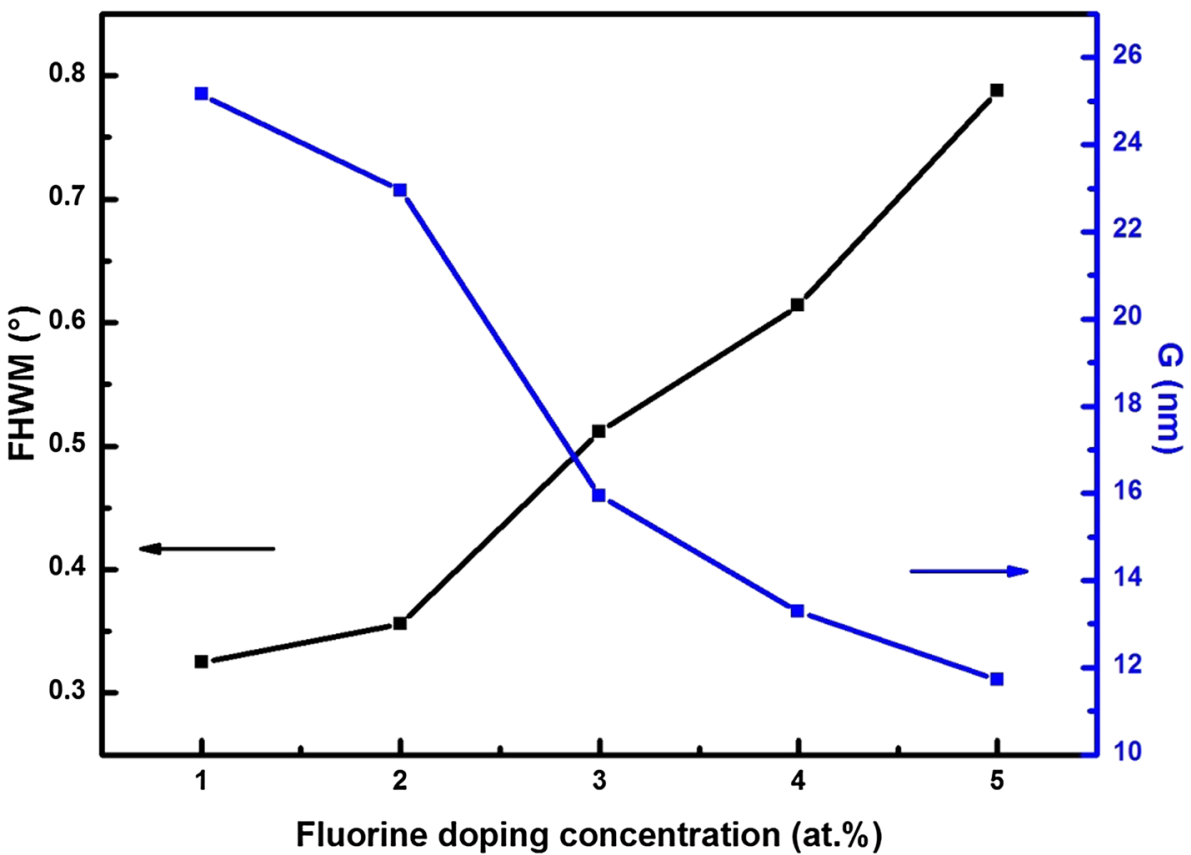


Fig. 3 TEM images of $\mathrm{SnO}_{2}$ nanoparticles: undoped $\mathrm{SnO}_{2}$ (a), FTO 3\% (b), and FTO 5\% (c). HR-TEM images of the $\mathrm{SnO}_{2}$ : undoped $\mathrm{SnO}_{2}(\mathbf{d})$ and FTO $3 \%$ (e)
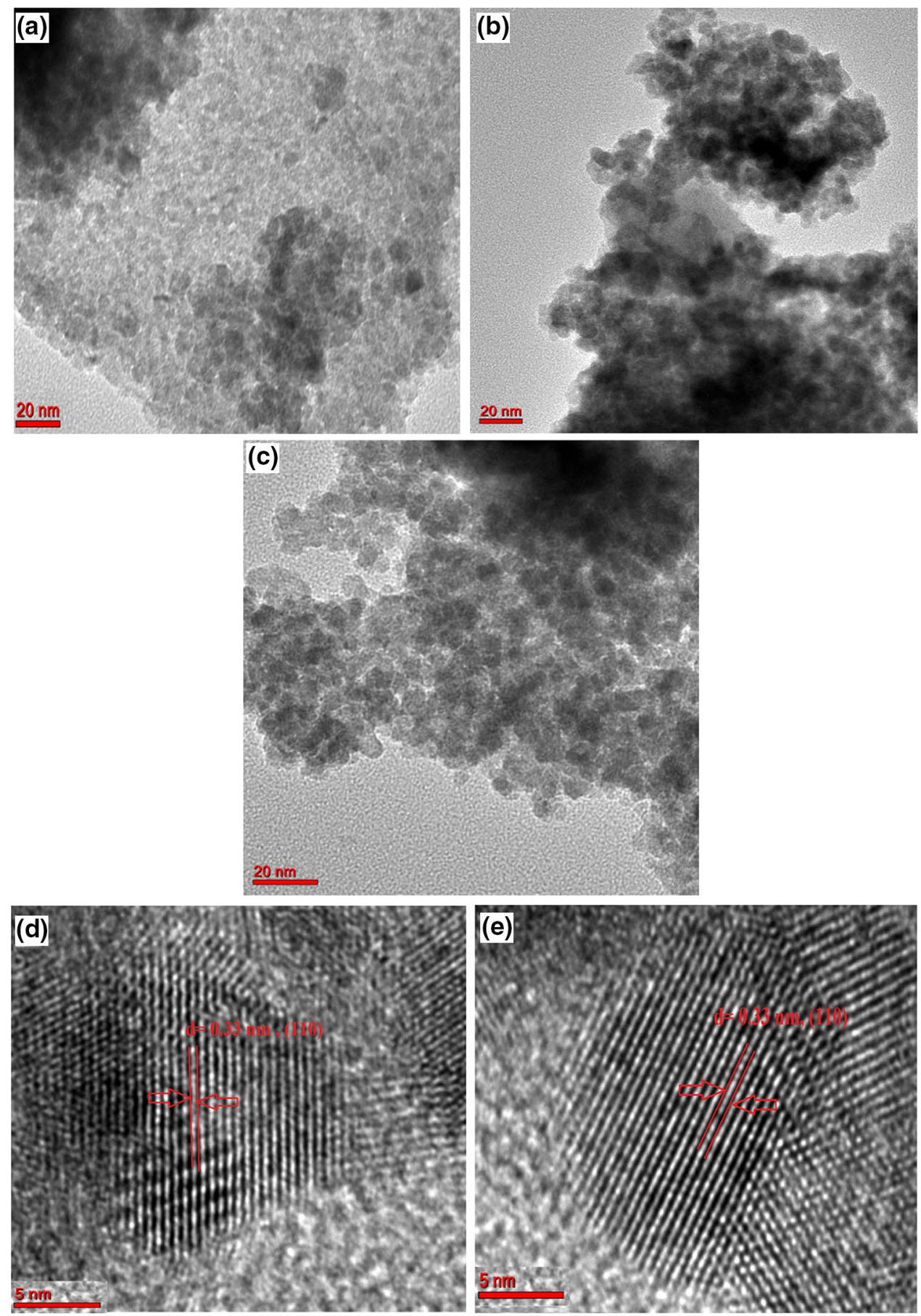

peak progressively shifted towards lower diffraction angle values compared to the peak position observed in the undoped sample, and broadened as the fluorine content was increased (Table 1; Fig. 1). These results indicate an increase in the lattice disorder due the strain induced by $\mathrm{F}^{-}$substitution [26].

The mean grain size of nanoparticles was estimated from Scherrer's formula $[27,28]$ :

$$
G=\frac{0.9 \lambda}{\beta \cos \theta}
$$

where $\lambda$ is the $\mathrm{X}$-ray wavelength, $\theta$ is the maximum of the Bragg diffraction peak (in radians) and $\beta$ is the full width at half maximum (FWHM) of the XRD peak. The average grain size ranged from 11 to $25 \mathrm{~nm}$. Indeed, for the undoped sample the crystallite size was about $23 \mathrm{~nm}$ and it increased rapidly to $25 \mathrm{~nm}$, for 1 at. $\% \mathrm{~F}$ doped $\mathrm{SnO}_{2}$, then 
it decreased with the raising of fluorine content as it was shown in Table 1. Figure 2 presents the variation of grain size and the FWHM with $\mathrm{F}$ doping concentration. It is clearly observed that, as the nanoparticles become smaller, the FWHM keeps increasing with the increase of F doping content. This indicates that the crystallinity of the powders decreases gradually depending on the dopant incorporation. On the other hand, we found that after the incorporation of $\mathrm{F}$, with the decrease in the particle size, a/c ratio increases from 1.486 to 1.498 suggesting the compressed state of the lattice (Table 1). Thus, it implies that all the doped samples exhibit compressive stresses, due to the substitution of $\mathrm{O}^{2-}$ by $\mathrm{F}^{-}$ion. Similar behavior was observed by Thirumoorthi et al. [29]. It is therefore concluded that the fluorine content strongly affects the structure of $\mathrm{SnO}_{2}$, as observed elsewhere [30].

To further study the morphology of these nanoparticles, TEM measurements combined with high-resolution TEM was employed. Figure 3 shows the TEM images of FTO nanoparticles with different doping levels. It's obvious that the particles are basically spherical in shape and in an agglomerated form by superposition of nanometric size particles. The TEM images also show that the particle size of undoped $\mathrm{SnO}_{2}$ is smaller than $50 \mathrm{~nm}$. However, further increase of fluorine content, will lead to smaller grain size than that of the undoped sample (Fig. 3b, c). It can be therefore concluded that $\mathrm{F}$ doping improves the growing up of nanosized tin oxide. These observations confirm the size of $\mathrm{SnO}_{2}$ nanoparticles calculated from the Scherrer's equation (Table 1) which indicates that the nanoparticles are well-crystallized in all cases. The HR-TEM images of undoped and fluorine doped $\mathrm{SnO}_{2}$ are also shown in Fig. 3, confirming the presence of highly crystalline rutile-type $\mathrm{SnO}_{2}$. The well-resolved lattice fringes (Fig. 3d, e) show that the spacing is about $0.33 \mathrm{~nm}$ corresponding to the (110) plane of the rutile crystal structure of the nanocrystalline $\mathrm{SnO}_{2}$. In order to confirm the chemical composition of FTO nanoparticles, EDS analysis was performed. The results reported in Table 2 confirmed the presence of fluorine in the $\mathrm{SnO}_{2}$ matrix with no other impurities. This means that FTO nanoparticles were homogeneous and quasi-stoichiometric.

Table 2 Atomic compositions of FTO nanoparticles

\begin{tabular}{llll}
\hline at.\% F & $\mathrm{O}($ at.\%) & Sn (at.\%) & $\mathrm{F}($ at.\%) \\
\hline 0 & 69.21 & 30.79 & 0 \\
1 & 67.86 & 30.66 & 1.48 \\
2 & 66.13 & 31.86 & 2.01 \\
3 & 65.28 & 31.60 & 3.12 \\
4 & 65.19 & 30.61 & 4.20 \\
5 & 64.77 & 29.90 & 5.33 \\
\hline
\end{tabular}

Raman spectroscopy is a useful tool for the characterization of nanosized materials and is a qualitative probe of the presence of lattice defects in solids. Figure 4 presents the typical room temperature Raman spectra of the assynthesized undoped and $\mathrm{F}$ doped $\mathrm{SnO}_{2}$ (3 at.\%). Three fundamental Raman peaks are observed at 477,632 , and $782 \mathrm{~cm}^{-1}$, corresponding to the $\mathrm{E}_{\mathrm{g}}, \mathrm{A}_{1 \mathrm{~g}}$, and $\mathrm{B}_{2 \mathrm{~g}}$ vibration modes, respectively, in a good agreement with those for the rutile $\mathrm{SnO}_{2}$ single crystal [31-33]. It is noted that the $\mathrm{A}_{1 \mathrm{~g}}$ mode of $\mathrm{F}$ doped $\mathrm{SnO}_{2}$ is more broadened than that of the undoped sample, which is induced by the size effect of crystallites. In addition to the fundamental Raman peaks of rutile $\mathrm{SnO}_{2}$, there are other peaks observed at 297 and $366,710 \mathrm{~cm}^{-1}$ corresponding to IR-active $\mathrm{E}_{\mathrm{u}} \mathrm{TO}$ and $E_{u}$ LO modes, respectively. On the other hand, the Raman mode $A_{2 g}$ is observed only at $410 \mathrm{~cm}^{-1}[34,35]$ in the case of FTO nanoparticles, may be due to the doping effect. These additional Raman peaks are similar to the Raman spectra of $\mathrm{SnO}_{2}$ nanopowder reported previously [36].

Absorption spectroscopy was used to explore the effect of the synthesis method and the synthesis conditions on the optical properties of $\mathrm{SnO}_{2}$ nanoparticles. It is well known that theory of optical absorption gives the relationship between the absorption coefficients $\alpha$ and the photon energy $h \nu$ for direct allowed transition as [37]:

$(\alpha h v)^{2}=C\left(h v-E_{g}\right)$

where $E_{g}$ is the apparent optical band gap and $C$ is a constant characteristic of the material. The direct band gap is determined using this equation when the straight portion of the $(\alpha h \nu)^{2}$ against $h \nu$ plot is extrapolated to intersect the energy axis at $\alpha=0$. Figure 5 shows the plots of $(\alpha h \nu)^{2}$ versus photon energy $h \nu$ for FTO nanoparticles with

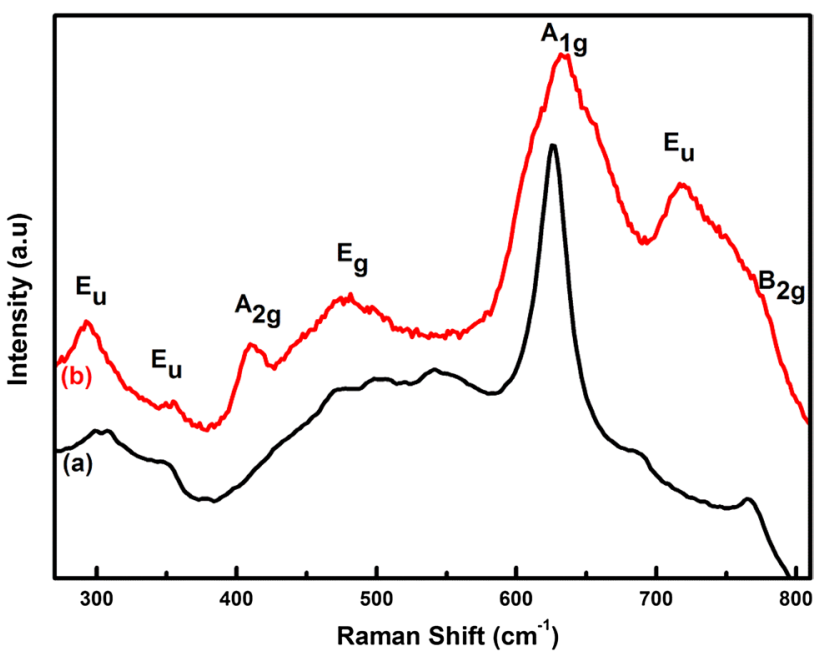

Fig. 4 Room-temperature Raman spectra of FTO nanoparticles: undoped $\mathrm{SnO}_{2}(a)$ and 3 at. $\% \mathrm{~F}$ doped $\mathrm{SnO}_{2}(b)$ 


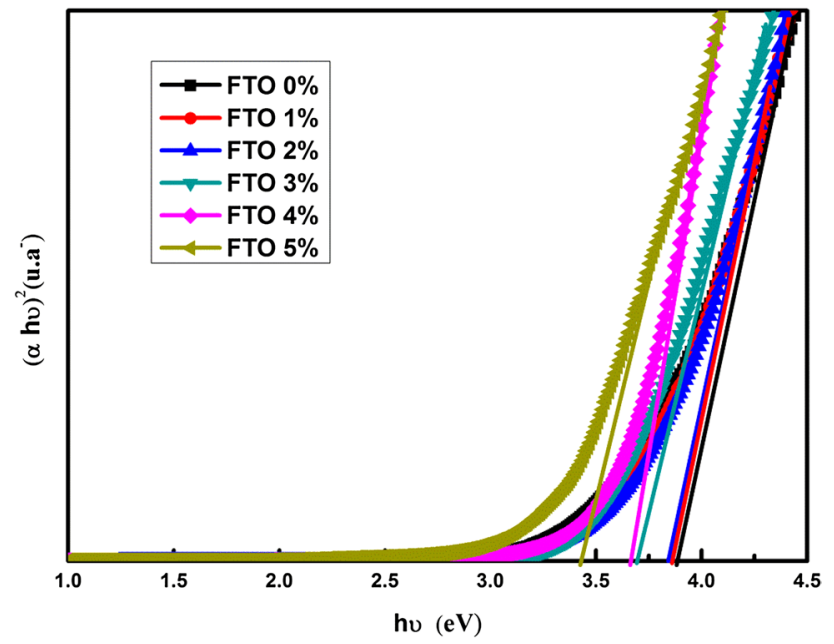

Fig. 5 Plots of $(\alpha h \nu)^{2}$ versus photon energy $h \nu$ of FTO nanoparticles
Table 3 Band gap $\left(\mathrm{E}_{\mathrm{g}}\right)$ and Urbach energy $\left(\mathrm{E}_{\mathrm{u}}\right)$ of samples with different fluorine doping concentrations

\begin{tabular}{lll}
\hline FTO (at.\%) & $\mathrm{E}_{\mathrm{g}}(\mathrm{eV})$ & $\mathrm{E}_{\mathrm{u}}(\mathrm{eV})$ \\
\hline 0 & 3.881 & 0.634 \\
1 & 3.872 & 0.645 \\
2 & 3.854 & 0.652 \\
3 & 3.701 & 0.755 \\
4 & 3.672 & 0.777 \\
5 & 3.444 & 0.805 \\
\hline
\end{tabular}

different doping concentrations. The linear part of the plot has been extrapolated towards energy axis. The estimated band gap values are listed in Table 3. The values obtained for the band gap energies of the FTO vary between 3.88 and $3.44 \mathrm{eV}$ and are in good agreement with other reported values $[38,39]$. The band gap energies of the $\mathrm{SnO}_{2}$ materials show a decrease with the increase of doping concentration (Fig. 6).

The band gap of undoped nanoparticles was found to be equal to $3.8 \mathrm{eV}$ which is higher than that of bulk $\mathrm{SnO}_{2}$ $(3.6 \mathrm{eV})$. This increase in the band gap of both undoped and doped nanoparticles as compared to bulk can be ascribed to quantum confinement effect and the reduction of structural defects. This confirms the good crystalline quality of the nanopowder obtained by sol gel method.

According to Urbach effect; an increase of doping concentration would induce the formation of band tails in the band gap which is expressed by [40]:

$\alpha=\alpha_{0} \exp \left(\frac{h v}{E_{u}}\right)$

where $\alpha_{0}$ is a constant and $\mathrm{E}_{\mathrm{u}}$ is the Urbach energy, which refers to the optical transition between occupied states in the valence band tail and the conduction band edge. The $\mathrm{E}_{\mathrm{u}}$ values are obtained from the inverse of the slope of $\operatorname{Ln}(\alpha)$ versus photon energy. As shown in Fig. 6 and reported in Table 3 an increase of the disorder appears from the introduction of dopants $\left(0-5\right.$ at.\%) in the $\mathrm{SnO}_{2}$ lattice. This increase is due to increased acceptor levels of interstitial oxygen atoms.

Based on the previous results obtained, we deduced that 1 at.\% content is the most amount of fluorine doping. For that, we have chosen this sample to characterize its electrical property and study the strong correlation between the different properties.
Fig. 6 Evolution of the energy band gap $\left(\mathrm{E}_{\mathrm{g}}\right)$ and the Urbach energy $\left(E_{u}\right)$ depending on the dopant levels

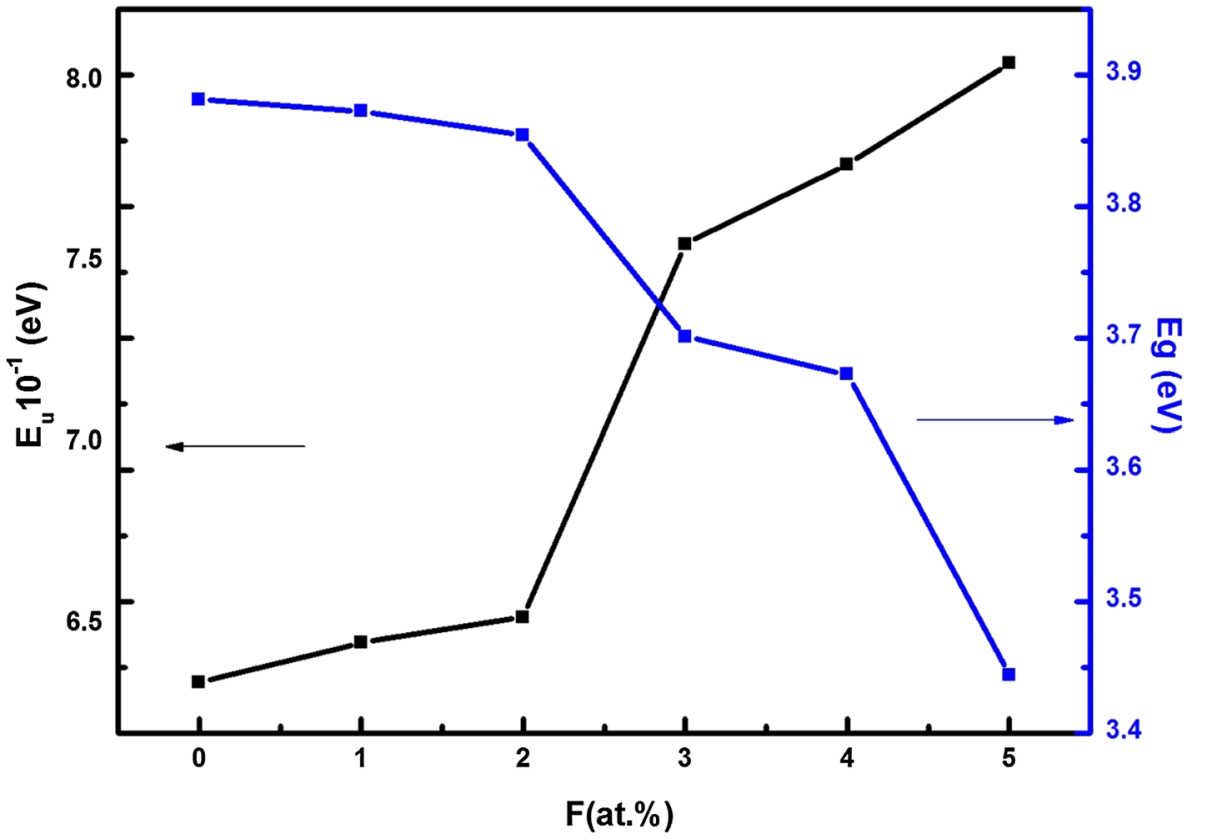


Impedance spectroscopy is a powerful method to characterize the electrical properties of materials. The conductivity of the polycrystalline system is led by various processes such as bulk conduction and grain boundary conduction, and the analysis of complex impedance spectra allows to specify the contribution of each process in the total conductivity $[41,42]$. The impedance spectrum usually represented as the imaginary component of the impedance $\left(-Z^{\prime \prime}\right)$ versus the real component $Z^{\prime}$, which is referred as Nyquist plot, is taken over a wide frequency range $(40 \mathrm{~Hz}-1 \mathrm{MHz})$ and shown in Fig. 7. These complex impedance measurements were carried out for undoped $\mathrm{SnO}_{2}$ and FTO 1 at.\% at different temperatures. The plots have the shape of semicircles arcs, indicating that the grains are homogenous in size for different doping rates [43]. It can be seen from Fig. 7a, the existence of semicircles which appear only at temperatures up to $420 \mathrm{~K}$, while in the case of FTO 1 at.\% sample, the semicircles became clear from $300 \mathrm{~K}$ (Fig. 7b). This can be explained by the enhancement of the conductivity with the temperature and doping. A conductivity threshold is required to extract the signal from the noise. On other hand, a reduction of the semicircles diameters size is observed with the increase in temperature; this reduction reflects the decrease in the resistance value and so a semiconducting character of the material [44]. This phenomenon is mainly assigned to the dependence of the charge carrier mobility with temperature, the conductivity increases with temperature increase.

It is well known that the complex impedance analysis permits the correlation between the microstructure of the sample and its electrical properties. Usually, the material is modeled by an electrical equivalent circuit. According to the complex impedance plots shown in Fig. 7, all samples can be modeled by an electrical circuit composed by a grain resistance $\mathrm{Rg}$ connected in series with a parallel combination grain boundaries resistance $\mathrm{Rgb}$ and capacitance $\mathrm{Cgb}$.

The frequency dependent electrical properties of a material are normally described in terms of its conductivity $(\sigma)$ which has been calculated from the conductance $\mathrm{G}$ by using the relation:

$\sigma=\frac{e}{G \times S}$

where e/S represents the geometrical sample ratio.

Figure 8a, b show the variation of the conductivity with frequency of $\mathrm{SnO}_{2}$ and FTO 1 at.\% at different temperatures, respectively. The frequency dependence of the conductivity is characterized by the occurrence of a frequency independent region for each temperature. In the low frequency region, a plateau is observed, where the conductivity is frequency independent. But in the higher frequency region $(f>10 \mathrm{kHz})$, it increases with increasing frequency; this region is called "a dispersive region". In such region,

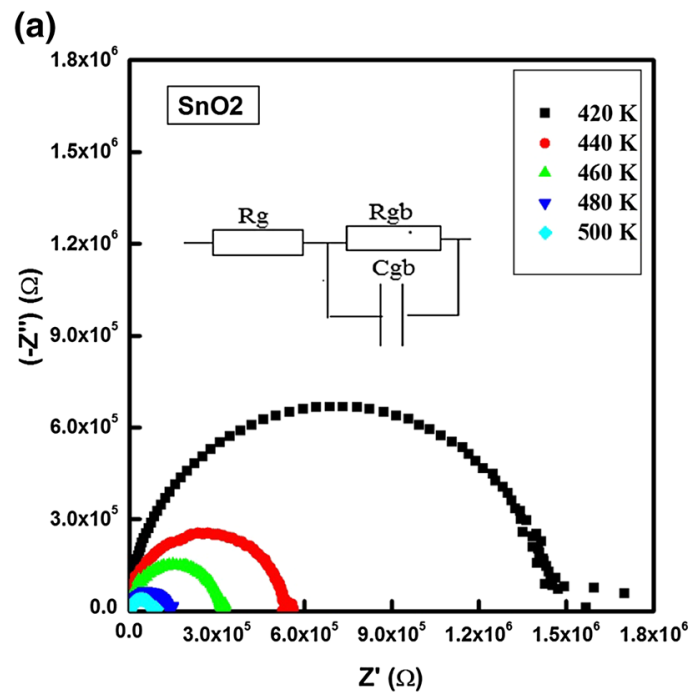

(b)

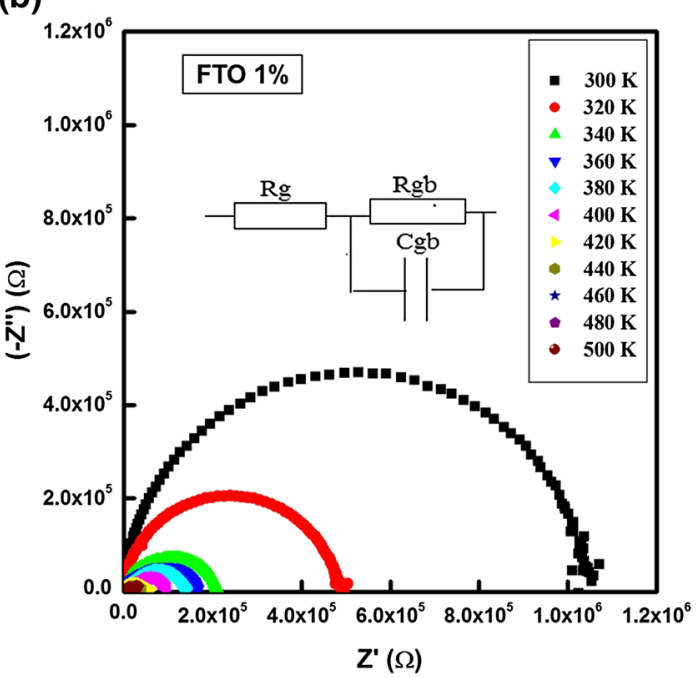

Fig. 7 a, b Experimental Nyquist diagrams of $\mathrm{SnO}_{2}$ and FTO $1 \%$ samples measured at different temperature

the frequency dependence can be described by the Jonscher's power law [45]:

$\sigma \propto A \omega^{s}$

where $A$ is a pre-exponential factor and $s$ is the frequency exponent which depends on temperature.

The deduced $s$ values from fits using Eq. (7), in the high frequency region, for different measurement temperatures, are depicted in Fig. 9a, b. The obtained values indicate that $0<\mathrm{s}<1$, characterizing the probable dominance of hopping conduction mechanism $[45,46]$ and proving that process is thermally activated. Also, the $s(\mathrm{~T})$ curve is in good agreement with Mott law:

$\mathrm{s}=1-\left(6 \mathrm{k}_{\mathrm{B}} \mathrm{T} / \mathrm{W}_{\mathrm{m}}\right)$ 


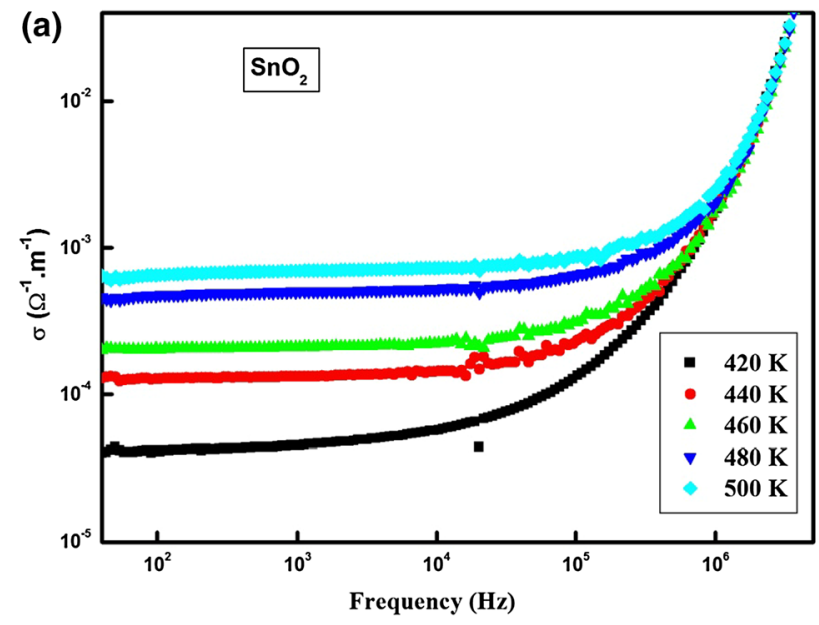

(b)

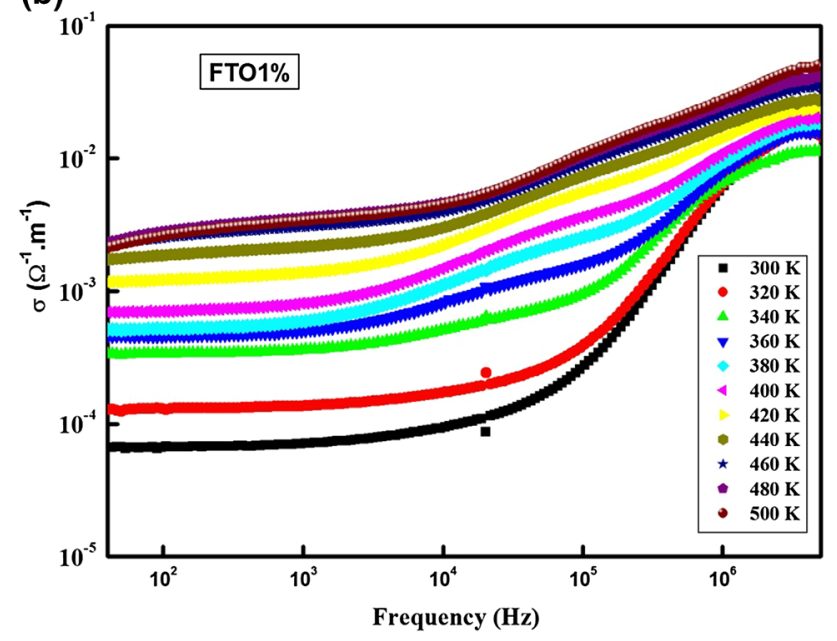

Fig. 8 a, b Variation of the conductivity of different FTO samples versus frequency

where $\mathrm{W}_{\mathrm{m}}$ is the binding energy, which is defined as the energy required to remove an electron completely from one site to another site and $\mathrm{k}_{\mathrm{B}}$ is Boltzmann's constant.

For the same temperature, we show that the conductivity of the FTO 1 at.\% sample is higher than that of $\mathrm{SnO}_{2}$ one. This increase in conductivity can be related to the increase of the grain size and the substitution of $\mathrm{O}^{2-}$ ions by the $\mathrm{F}^{-}$doping ions.

To sum up, doping affects well on the electrical properties of material that it improves the conductivity and impedance of the sample. This may be explained as follow: when fluorine is incorporated in tin oxide, each $\mathrm{F}^{-}$anion substitutes an $\mathrm{O}^{2-}$ anion in the lattice and the substituted $\mathrm{O}^{2-}$ anion introduces more free electrons. This results in an increase in free electrons with fluorine doping. Moreover, the $\mathrm{F}$ atoms that occupy the proper lattice positions contribute to enhance the disorder (grain boundary) of the structure and grain size which increase the charge carrier mobility.
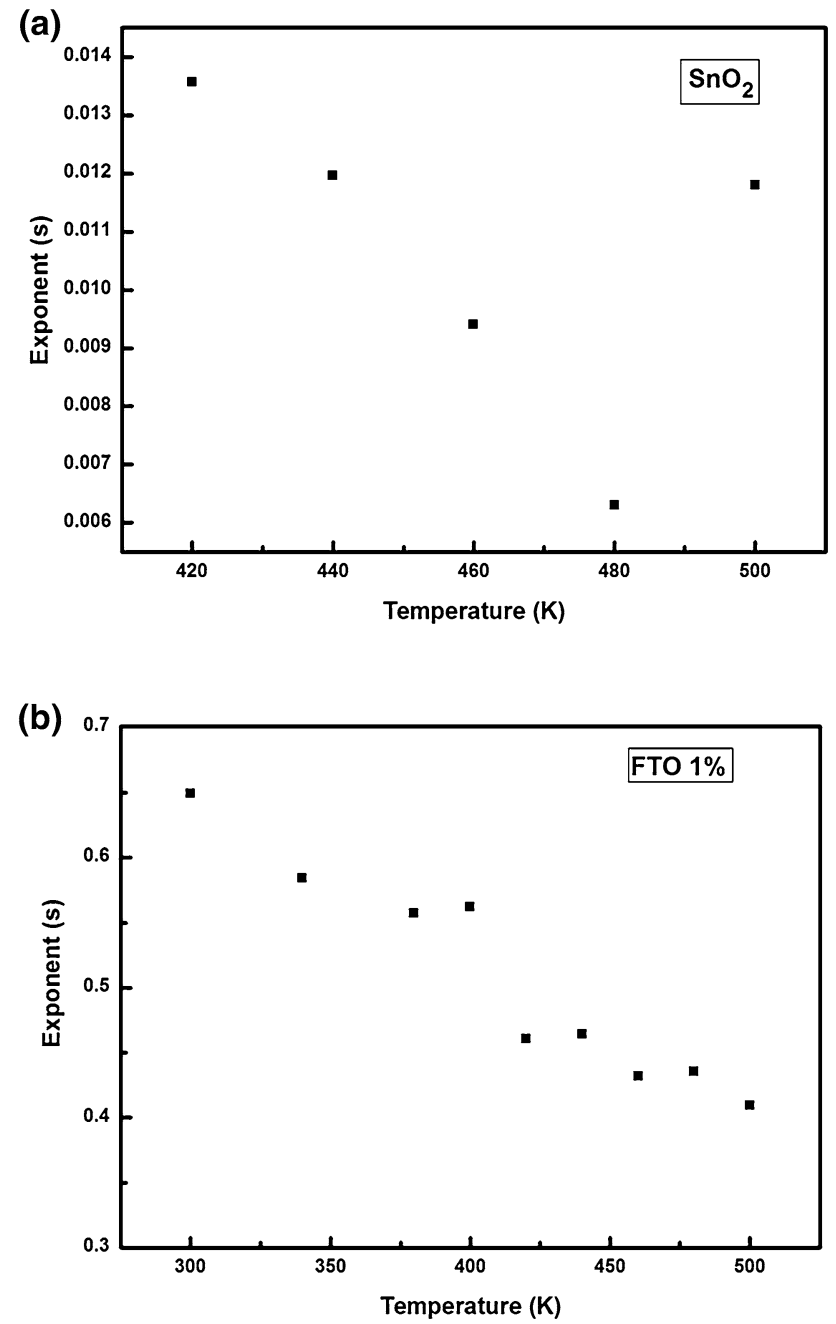

Fig. 9 a, b Exponent $s$ versus measurement temperature of the synthesized $\mathrm{SnO}_{2}$ and FTO 1\% nanoparticles

\section{Conclusion}

In the present study, Fluorine doped $\mathrm{SnO}_{2}$ aerogel nanoparticles were successfully synthesized by using a relatively simple sol-gel method followed by a drying in the supercritical conditions of ethyl alcohol. The effect of Fluorine doping on the structural, morphological, optical and electrical properties of $\mathrm{SnO}_{2}$ was investigated. XRD studies reveal that the samples exhibit good crystallinity with a tetragonal structure. The grain size of as-synthesized FTO is calculated to be $11-25 \mathrm{~nm}$ from XRD and TEM patterns. The optical band gap of FTO nanoparticles depends on F doping and varies from 3.88 to $3.44 \mathrm{eV}$. Impedance spectroscopy and conductivity results indicate that the electrical properties are strongly dependent on temperature and doping. It was concluded that the crystallinity and optical properties of the $\mathrm{SnO}_{2}$ nanomaterials depend strongly on Fluorine doping rate. This work has shown also the ability to 
grow FTO nanoparticles with lower levels of doping using a cost effective and environmentally friendly method.

Open Access This article is distributed under the terms of the Creative Commons Attribution 4.0 International License (http:// creativecommons.org/licenses/by/4.0/), which permits unrestricted use, distribution, and reproduction in any medium, provided you give appropriate credit to the original author(s) and the source, provide a link to the Creative Commons license, and indicate if changes were made.

\section{References}

1. L. Tan, L. Wang, Y. Wang, J. Nanomater 10 (2011)

2. N. Talebian, F. Jafarinezhad, Ceram. Int. 39, 8311 (2013)

3. D.S. Ginley, Handbook of transparent conductors. (Springer, New York, 2010)

4. Ç. Kılıç, A. Zunger, Phys. Rev. Lett. 88, 095501 (2002)

5. R.D. Sakhare, G.D. Khuspe, S.T. Navale, R.N. Mulik, M.A. Chougule, R.C. Pawar, C.S. Lee, S. Sen, V.B. Patil, J. Alloys Compd. 563, 300 (2013)

6. A.A. Zhukova, M.N. Rumyantseva, V.B. Zaytsev, A.V. Zaytseva, A.M. Abaku-mov, A.M. Gaskov, J. Alloys Compd. 565, 6 (2013)

7. S. Balaji, R. Vasuki, D. Mutharasu, J. Alloys Compd. 554, 25 (2013)

8. C. Karunakaran, S. Sakthi Raadha, P. Gomathisankar, J. Alloys Compd. 549, 269 (2013)

9. E. Elangovan, K. Ramamurthi, Appl. Surf. Sci. 249, 183 (2005)

10. Z.Y. Banyamin, P.J. Kelly, G. West, J. Boardman, Coatings 4, $732(2014)$

11. C.H. Han, S.D. Han, S.P. Khatkar, Sensors 6, 492 (2006)

12. C.H. Han, D.U. Hong, J. Gwak, S.D. Han, Korean J. Chem. Eng. 24, 927 (2007)

13. S. Chaisitsak, Sensors 11, 7127 (2011)

14. V. Kumar, A. Govind, R. Nagarajan, Inorg. Chem. 50, 5637 (2011)

15. Z. Tachan, S. Rühle, A. Zabann, Sol. Energy Mater. Sol. Cells 94, 317 (2010)

16. I.Y.Y. Bu, Ceram. Int. 40, 417 (2014)

17. H.L. Zhao, Q.Y. Liu, Y.X. Cai, F.C. Zhang, Mater. Lett. 62, 1294 (2008)

18. S. Wu, S. Yuan, L. Shi, Y. Zhao, J. Fang, J. Colloid Interface Sci. 346, 12 (2010)

19. G.E. Patil, D.D. Kajale, V.B. Gaikwad, G.H. Jain. Int. Nano Lett. 2, 17 (2012)

20. E. Elangovan, M.P. Singh, K. Ramamurthi, Mater. Sci. Eng. B 113, 143 (2004)
21. Q.P. Tran, J.S. Fang, T.S. Chin, Mater. Sci. Semicond. Process. 40, 664 (2015)

22. Zhong X, Yang B, Zhang X, Jia J, Yi G, Particuology 10, 365 (2012)

23. A. Gamard, O. Babot, B. Jousseaume, M.C. Rascle, T. Toupance, G. Campet, Chem. Mater. 12, 3419 (2000)

24. Y. Ren, G. Zhao, Y. Chen, Appl. Surf. Sci. 258, 914 (2011)

25. E. Abdelkader, L. Nadjia, B. Naceur, B. Noureddine, J. Alloys Compd. 679, 408 (2016)

26. H. Kim, G.P. Kushto, R.C.Y. Auyeung, A. Piqué, Appl. Phys. A 93, 521 (2008)

27. B.D. Cullity, Elements of X-ray diffraction. (Addison-Wesley, Reading, 1978), p. 102

28. D.L. Chen, L. Gao, J. Colloid Interface Sci. 279, 137 (2004)

29. M. Thirumoorthi, J. Thomas, J. Prakash, Superlattices Microstruct. 89, 378 (2016)

30. V. Bilgin, I. Akyuz, E. Ketenci, S. Kose, F. Atay, Appl. Surf. Sci. 256, 6586 (2010)

31. J.F. Scott, J. Chem. Phys. 53, 852 (1970)

32. H. Kohno, T. Iwasaki, Y. Mita, S. Takeda, J. Appl. Phys. 91, $3232(2002)$

33. V.G. Kravets, Opt Spectrosc. 103, 766 (2007)

34. R.S. Katiyar, P. Dawson, M.M. Hargreave, G.R. Wilkinson, J. Phys. C 4, 2421 (1971)

35. P.S. Peercy, B. Morosin, Phys. Rev. B 7, 2779 (1973)

36. A. Ayeshamariam, S. Ramalingam, M. Bououdina, M. Jayachandran, Spectrochim. Acta Part A 118, 1135 (2014)

37. J.I. Pankove, Optical processes in semiconductors. (Dover, New York, 1976), p. 91

38. Lei Li, C. Zhang, W. Chen, Nanoscale 7, 12133 (2015)

39. F. de Moure-Flores, A. Guillén-Cervantes, K.E. Nieto-Zepeda, J.G. Quiñones-Galván, A. Hernández-Hernández, M. de la Olvera, M. Meléndez-Lira, Rev. Mex. Fis. 59, 335 (2013)

40. S. Aksoy, Y. Caglar, S. Ilican, M. Caglar, J. Alloys Compd. 512, 171 (1992)

41. E. Barsoukov, J.R. Macdonald (eds.), Impedance spectroscopy: theory, experiment, and applications, 2nd edn. (Wiley, Hoboken, 2005)

42. A.R. West, D.C. Sinclair, N. Hirose, J. Electro Ceram. 1, 65 (1997)

43. M.A. Batal, F.H. Jneed, Energy Procedia 6, 1 (2011)

44. A.R. Babara, S.B. Kumbhara, S.S. Shindea, A.V. Moholkara, J.H. Kimb, K.Y. Rajpure, J. Alloys Compd. 509, 7508 (2011)

45. A.K. Jonscher, Nature 276, 673 (1977)

46. H. Bottger, U.V. Bryskin, Hopping conduction in solids. (Verlag Akademie, Berlin, 1985) 